\title{
Concerning the Landau Pole in 3-3-1 Models
}

\author{
Alex G. Dias* \\ Instituto de Física, Universidade de São Paulo, \\ C. P. 66.318, 05315-970 São Paulo, SP, Brazil. \\ R. Martinez ${ }^{\dagger}$ \\ Departamento de Física, \\ Universidad Nacional de Colombia \\ Bogotá, Colombia. \\ V. Pleitez \\ Instituto de Física, Teórica, Universidade Estadual Paulista \\ Rua Pamplona 145, São Paulo, SP, Brazil.
}

(Dated: October 9, 2018)

\begin{abstract}
Some 3-3-1 models predict the existence of a non-perturbative regime at the TeV scale. We study in these models, and their supersymmetric extensions, the energy at which the non-perturbative limit and a Landau-like pole arise. An order of magnitude for the mass of the extra neutral vector boson, $Z^{\prime}$, present in these models is also obtained.
\end{abstract}

PACS numbers: $12.60 . \mathrm{Cn}, 12.60 . \mathrm{Jv}$

\footnotetext{
* e-mail: alexdias@fma.if.usp.br

$\dagger$ e-mail: remartinezm@unal.edu.co

$\ddagger$ e-mail: vicente@ift.unesp.br
} 


\section{INTRODUCTION}

The so called 3-3-1 extensions of the standard model (SM) are interesting options for the physics at the TeV scale [1, 2]. Although these models coincide at low energies with the SM, they explain some fundamental questions that are accommodated, but not explained by the former. For instance, i) in order to cancel chiral anomalies the number of generation $N_{g}$ must be a multiple of 3, but because of the asymptotic freedom in QCD, which implies that the number of generations must be $N_{g} \leq 5$, it follows that in those models the only number of generations allowed is $N_{g}=3$; ii) these models as any with $S U(3)_{W}$ symmetry explain why $\sin ^{2} \theta_{W}<1 / 4$ at the $Z$-pole (see below); iii) the electric charge is quantized independently of the nature of neutrinos [3]; iv) the Peccei-Quinn symmetry is almost an automatic symmetry of the classical Lagrangian [4] and with a minimal modification, the PQ symmetry as the solution to the strong CP problem is automatically implemented and the axion is protected against semiclassical gravity effects [5]; v) the theory becomes non-perturbative at the $\mathrm{TeV}$ scale, and the same happens with the respective $\mathcal{N}=1$ supersymmetric version [6]. There are other models with $S U(3)_{W}$ symmetry [7], but some of them imply charged heavy bileptons which are stable, that in turn lead to potentially cosmological troubles [8]. Another interesting possibility to consider is by introducing extra dimensions [9] and the orbifold compactification [10, 11], or other sort of 3-3-1 models as in Refs. 12]. A common feature of models with $S U(3)_{W}$ electroweak symmetry is the existence of simply and doubly charged or neutral real or/and non-hermitian vector bósons. For instance, the doubly charged and the real neutral vector bosons can be discovered by measurement of the left-right asymmetries in lepton-lepton scattering [13, 14], in muonium-antimuonium transitions [15, 16] or in accelerator processes [17, 18].

One of the main feature of these models is the fact that when the $g_{L}$ and $g_{X}$ coupling constants of the gauge groups $S U(3)_{L}$ and $U(1)_{X}$, respectively, are related with the electroweak mixing angle, the following relation is obtained

$$
\frac{g_{X}^{2}}{g_{L}^{2}}=\frac{\sin ^{2} \theta_{W}}{1-4 \sin ^{2} \theta_{W}}
$$

in the models of Refs. [1, 2]. When $\sin ^{2} \theta_{W}(\mu)=1 / 4$ the coupling constant $g_{X}(\mu)$ becomes infinite, i.e., a Landau-like pole arises, however the theory loses its perturbative character even at an energy scale lower than $\mu$. The other possibility $g_{L} \rightarrow 0$ is ruled out since $g_{L}$ is 
the same as in the standard model, $g_{2}$, due to the fact that the $S U(2)_{L}$ subgroup is totally embedded into $S U(3)_{L}$.

The possible existence of a Landau-like pole in 3-3-1 models is not unexpected since every non-assintotically free theory seems to has such a behavior. The new feature is that in some of these models that behavior may happens at energies of just few TeVs. This will imply that the cut-off, $\Lambda_{\text {cutoff }}$, in the theory can not be eliminated, by taking $\Lambda_{\text {cutoff }} \rightarrow \infty$, as it is expected in renormalizable theories. In this limit the theory might be a trivial theory. This is supposed to be the case of pure QED since the works of Landau and co-workers [19]. From the phenomenological point of view this result is not very dangerous, we already know that QED has to be embedded in the electroweak theory at a few hundred GeVs, and also that weak and strong correction have to be taken into account in the calculations of physical observables, even those that are purely electromagnetic in origin, like the $(g-2)_{\mu}$ factor, etc.

However, as a mathematical laboratory it is interesting to study pure QED at arbitrary small distances. In fact, lattice calculation suggest that chiral symmetry breaking allows QED to escape the Landau pole problem [20, 21]. This should happen because the chiral symmetry breaking is always strong enough to push the Landau pole above the cutoff [20]. It is interesting that the possibility of the existence of the Landau pole, or that the theory is a trivial one, arises already at the lower order in perturbation theory. More sophisticated calculation only enhance our confidence that this phenomenon is not an effect of the perturbation theory at lowest order. This is in accord with the point of view that the renormalization group provide qualitative guidance with respect to the asymptotic behavior at very high energies even where coupling constant at the scale of interest are too large to allow the use of perturbation theory. In particular this method provides usual insight into the types of possible behavior in field theories [22].

Notwithstanding, we must remember that both QED and the standard model are effective, and not fundamental, theories. It means that effective operators with dimensions higher that $d=4$ have to be considered if we want, for instance, to get a realistic continuum limit in lattice calculations [22]. Thus it seem that using the pure versions of these models are still inconclusive and the renormalization group may give an insight in this issue in 3-3-1 models.

The possibility of triviality implies that new phenomena must enter before the reach the 
Landau pole: or a new phase of the theory or the theory must be embedded in a more general one. This has been used in the context of the standard model to constraint the upper value of the Higgs boson mass [23]. Moreover, recently it has been argued that this upper limit on the Higgs bosons mass does not come from an instability of the vacuum [24].

In the past years some authors, using perturbation theory, have calculated the energy scale at which the weak mixing angle get the 0.25 value [25, 26, 27]. They have been found, taking into account only the degrees of freedom of the standard model, that this condition occurs at an energy scale of the order of $3-4 \mathrm{TeV}$ in the model of Ref. [1]. This value is an upper limit of the energy scale at which the Landau-like pole occurs (see the discussion in Sec. VI).

Our goal in this paper is to study the running of $\sin ^{2} \theta_{W}$ with energy in 3-3-1 models [1, 2] and their supersymmetric extensions given in Refs. [6]. However, since we have verified that, with the representation content of the minimal models $g_{L}$ does not change significatively, we study the running of $g_{X}$. We confirm the order of magnitude of the results of the previous works but we considered a more general scenario, when the $S U(3)_{L}$ symmetry breakdown scale, $\mu_{331}$, is inside the perturbative range and when the exotic quarks or SUSY particles are considered much heavier than the other particles. As previous calculations, ours are also done at the 1-loop level but we briefly comment the 2-loop case. For this reason our result have to be seen as an estimative of the energy scale where $\sin ^{2} \theta_{W}=0.25$. Using of perturbation theory to find a singularity could appears self-contradictory however, we recall that this behavior at relatively low energy, arises because of the constraint in Eq. (11). We think that our calculations as the previous ones in 3-3-1 models are only preliminary results. When lattice calculations, or other more appropriate techniques, were available they could be compared with those 1-loop calculations, as is usually done in the $\lambda \phi^{4}$ or QED cases [24].

The outline of this paper is as follows. In Sec. II] we revise briefly the minimal representation content of two 3-3-1 models that will be considered next. In Sec. III we give the evolution equation and calculate the $b_{i}$ coefficients in each model with and without supersymmetry. In this section we also reproduce the calculations of Refs. 25, 26, 27] of the energy at which the condition $s_{W}^{2}=0.25$ is satisfied, taking into account only the degrees of freedom of the standard model. In Sec. IV we study the evolution of $\alpha_{X} \equiv g_{X}^{2} / 4 \pi$, and calculate the energy scale, $M^{\prime}$, at which $\alpha_{X}\left(M^{\prime}\right)>1$. We also compare this energy with $\Lambda$ defined as $\alpha_{X}(\Lambda)=\infty$, taking into account the degrees of freedom of the 3-3-1 models 
for energies above an energy scale that we denote $\mu_{331}$. The last section is devoted to our conclusions.

\section{3-3-1 MODELS WITH DOUBLY CHARGED VECTOR BOSONS}

The models that we will take into account in this section are characterized by the electric charge operator,

$$
\mathcal{Q}_{A}=\frac{1}{2}\left(\lambda_{3}-\sqrt{3} \lambda_{8}\right)+X
$$

with two different representation content in the leptonic sector that are giving either by $\Psi_{a L}=\left(\nu_{a}, l_{a}, E_{a}^{+}\right)_{L}^{T} \sim(\mathbf{1}, \mathbf{3}, 0)$ (Model A) or by $\Psi_{a L}=\left(\nu_{a}, l_{a}, l_{a}^{c}\right)_{L}^{T} \sim(\mathbf{1}, \mathbf{3}, 0)$ (Model B), $a=e, \mu, \tau$. Both models contain doubly charged vector bosons. In the first case, we have to add singlets $l_{a R} \sim(\mathbf{1}, \mathbf{1},-1), E_{a R} \sim(\mathbf{1}, \mathbf{1},+1)$; and neutrinos $\nu_{a R} \sim(\mathbf{1}, \mathbf{1}, 0)$, if necessary. In the second case only right-handed neutrinos have to be added, also if necessary. However, since neutral singlet representations do not affect the running of the coupling constants, we will not worry about them here. In both models the quarks transform as follows: $Q_{i L}=\left(d_{i}, u_{i}, j_{i}\right)_{L}^{T} \sim\left(\mathbf{3}, \mathbf{3}^{*},-1 / 3\right) ; i=1,2 ; Q_{3 L}=\left(u_{3}, d_{3}, J\right)_{L}^{T} \sim(\mathbf{3}, \mathbf{3}, 2 / 3)$, with the singlets $u_{\alpha R} \sim(\mathbf{1}, \mathbf{1}, 2 / 3), d_{\alpha R} \sim(\mathbf{1}, \mathbf{1},-1 / 3), \alpha=1,2,3, j_{i R} \sim(\mathbf{1}, \mathbf{1},-4 / 3)$, and $J_{R} \sim(\mathbf{1}, \mathbf{1}, 5 / 3)$,

In these models there are fields with masses of the order of magnitude of the $S U(3)_{L}$ energy scale. For instance, in Model A the scalar fields necessary to break the gauge symmetry down to $U(1)_{Q}$ and giving the correct mass to all fermions in the model are three triplets: $\eta=$ $\left(\eta^{0}, \eta_{1}^{-}, \eta_{2}^{+}\right)^{T} \sim(\mathbf{1}, \mathbf{3}, 0), \rho=\left(\rho^{+}, \rho^{0}, \rho^{++}\right) \sim(\mathbf{1}, \mathbf{3}, 1)$ and $\chi=\left(\chi^{-}, \chi^{--}, \chi^{0}\right) \sim(\mathbf{1}, \mathbf{3},-1)$.

We will denote the vacuum expectation values as follows: $\left\langle\eta^{0}\right\rangle=u / \sqrt{2},\left\langle\rho^{0}\right\rangle=v / \sqrt{2}$ and $\left\langle\chi^{0}\right\rangle=w / \sqrt{2}$. In Model B it is necessary to add an scalar sextet $S \sim(\mathbf{1}, \mathbf{6}, 0)$

$$
S=\left(\begin{array}{ccc}
\sigma_{1}^{0} & h_{1}^{-} & h_{2}^{+} \\
h_{1}^{-} & H_{1}^{--} & \sigma_{2}^{0} \\
h_{2}^{+} & \sigma_{2}^{0} & H_{2}^{++}
\end{array}\right)
$$

and we will use the notation $\left\langle\sigma_{2}^{0}\right\rangle=v_{2} / \sqrt{2}$. It is also possible to have $\left\langle\sigma_{1}^{0}\right\rangle \neq 0$ giving to the neutrinos a Majorana mass. We will not be concerned with this here.

In Model A the physical scalar spectra are such that a singly charged scalar, which is a 
linear combinations of $\eta_{1}^{+}$and $\rho^{+}$, has the square mass [28]

$$
M_{1+}^{2} \propto\left(u^{2}+v^{2}-\frac{f w v}{u}-\frac{f u w}{v}\right)
$$

and the other singly charged scalar is a linear combination of $\eta_{2}^{+}$and $\chi^{+}$and has a square mass

$$
M_{2+}^{2} \propto\left(w^{2}+v^{2}-\frac{f v w}{u}-\frac{f u v}{w}\right)
$$

where $f<0$ is the trilinear coupling with dimension of mass. We see that even if $f=0$ only one of the singly charged scalar has a low mass, the other one has a mass square proportional to $w^{2}$, so it is heavy enough (unless it is fine tuned) to be decoupled from low energy physics (below the breaking of the $S U(3)_{L}$ symmetry). The same happens with the doubly charged physical scalar, a linear combination of $\rho^{++}$and $\chi^{++}$, with a mass square

$$
M_{++}^{2} \propto\left(w^{2}+v^{2}-\frac{f u v}{w}-\frac{f u w}{v}\right)
$$

and we see that it is also too heavy (unless fine tuned) and it will not be considered at low energies. On the other hand, in the real scalar fields sector there are three physical scalar Higgs, two states that do not depend on $w$ and $f$ having square masses of the order of $u^{2}+v^{2}$ and the other one is heavy. It means that at low energies we consider two scalar doublets of $S U(2)_{L}$ when we use the standard model degrees of freedom. The vector bosons, $V^{-}$, $U^{--}$, the exotic quarks and scalar singlets, all of them may be heavy since their masses are proportional to $w$. The extra neutral vector boson $Z_{2}$, has an even higher mass and also it will not be considered at low energies. This vector bosons is a mixture of $Z$ and $Z^{\prime}$, but if we neglect this mixture $Z_{2} \approx Z^{\prime}$.

\section{THE EVOLUTION EQUATIONS}

The evolution equations of the coupling constants at the one loop level are given by

$$
\frac{1}{\alpha_{i}(M)}=\frac{1}{\alpha_{i}\left(M_{Z}\right)}+\frac{1}{2 \pi} b_{i} \ln \left(\frac{M_{Z}}{M}\right), i=1,2,3 ;
$$

where $\alpha_{i}=g_{i}^{2} / 4 \pi$ and $g_{3}, g_{2}, g_{1}$ are the coupling constant of the $S U(3)_{C}, S U(2)_{L}, U(1)_{Y}$ gauge groups, respectively. In the context of 3-3-1 models we define the $b_{i}$ coefficients cor-

responding to the coupling constants $g_{3}, g_{L}, g_{X}$ of the gauge groups $S U(3)_{C}, S U(3)_{L}, U(1)_{X}$, respectively. 
For a general $S U(N)$ gauge group the $b_{i}$ coefficients are given by

$$
b_{i}=\frac{2}{3} \sum_{\text {fermions }} T_{R i}(F)+\frac{1}{3} \sum_{\text {scalars }} T_{R i}(S)-\frac{11}{3} C_{2 i}(G)
$$

for Weyl fermions and complex scalars, and $T_{R}(I) \delta^{a b}=\operatorname{Tr}\left[T^{a}(I) T^{b}(I)\right]$ with $I=F, S$; $T_{R}(I)=1 / 2$ for the fundamental representation, $C_{2}(G)=N$ for $S U(N)$ and $C_{2}(G)=0$ for $U(1)$. For $U(1)_{y}$ we use $\sum T_{R 1}(F, S)=\sum y^{2}$ where $y=Y / 2$ for the standard model and $y=X$ for the 3-3-1 models. On the other hand for the respective supersymmetric version we have

$$
b_{i}^{\text {susy }}=\sum_{\text {fermions }} T_{R i}(F)+\sum_{\text {scalars }} T_{R i}(S)-3 C_{2 i}(G),
$$

and only the usual non-supersymmetric fields are counted now.

We will assume that the standard model with several scalar multiplets is valid until an energy scale $\mu_{331}, i$. e., below $\mu_{331}$ we consider the SM plus some light scalar doublets or triplets. A $S U(2)_{L} \otimes U(1)_{Y}$ model with $N_{g}=3$ fermion generations, $N_{H}$ scalar doublets $(Y=$ $\pm 1)$ and $N_{T}$ non-hermitian scalar triplets $(Y=2)$, using Eqs. (8) and the representation content above, implies

$$
\begin{aligned}
b_{1} & =\frac{1}{6} N_{H}+N_{T}+\frac{20}{3}, \\
b_{2} & =\frac{1}{6} N_{H}+\frac{2}{3} N_{T}-\frac{10}{3}, \\
b_{3} & =-7 .
\end{aligned}
$$

Notice that we have not used a grand unification normalization for the hypercharge $Y$ assignment. Since we will assume that $\mu_{\text {susy }} \approx \mu_{331}$ when considering the SUSY extensions of a 3-3-1 model, below $\mu_{331}$ the only effect of supersymmetry will be the addition of light scalar multiplets. Above $\mu_{331}$, we have to consider the degrees of freedom of the 3-3-1 models.

The heavy leptons, $E_{a}$ in Model A, quarks $J$ and $j_{i}$; the scalar singlets $\eta_{2}^{+}, \rho^{++}$; the scalar doublets like $\left(\chi^{-}, \chi^{--}\right)$; and finally, the vector doublets like $\left(V^{-}, Y^{--}\right)$and the extra neutral vector boson, $Z^{\prime}$, will not be considered as active degrees of freedom below $\mu_{331}$. Hence, in non-SUSY Model A we have $N_{H}=2$ and $N_{T}=0$; in the SUSY version $N_{H}=4$ and $N_{T}=0$. In the non-SUSY Model B we have to take into account the scalar sextet which implies an additional doublet and an non-hermitian triplet, so that $N_{H}=3$ and $N_{T}=1$, and $N_{H}=6$ and $N_{T}=2$ in the SUSY case. 
In the energy regime below $\mu_{331}$ we use the standard definition for $\sin ^{2} \theta_{W}(\mu)$ :

$$
\sin ^{2} \theta_{W}(\mu)=\frac{1}{1+\frac{\alpha_{2}(\mu)}{\alpha_{1}(\mu)}}, \quad \mu \leq \mu_{331}
$$

With this equation for the weak mixing angle and the $b_{i}$ coefficients in Eqs.(10), we obtain the value of $\sin ^{2} \theta_{W}\left(\mu_{331}\right)$ using the running equation of $\alpha_{1,2}^{-1}$ given by Eqs. (7), which will be used as an input for the case of energies above $\mu_{331}$. The values of the energies at which $\sin ^{2} \theta_{W}(\Lambda)=0.25$ (at which the curves cut the values 0.25 ), in models $\mathrm{A}$ and $\mathrm{B}$ with and without supersymmetry, are shown in Fig. 1. These energies give an order of magnitude of the energy scale of the Landau Pole. In particular, for the non-SUSY Model A (leftmost curve in the figure) we obtain a value for $\Lambda$ in agreement with that of Refs. 25, 26, 27], i.e., $\approx 4 \mathrm{TeV}$.

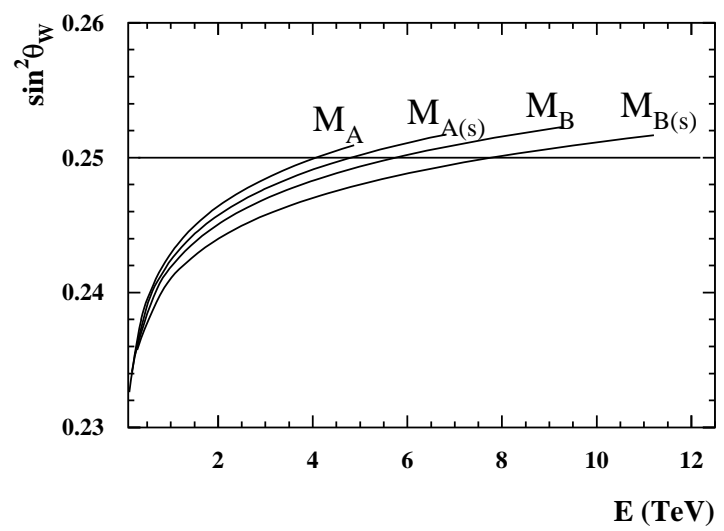

FIG. 1: Running of the electroweak mixing angle for Models A and B considering only the degrees of freedom of the effective 3-2-1 model. (s) stands for the respective supersymmetrized version.

Next, we consider the case of energies above the scale $\mu_{331}$. Now we have to use the relation

$$
\sin ^{2} \theta_{W}(\mu)=\frac{1}{4} \frac{1}{1+\frac{\alpha_{L}(\mu)}{4 \alpha_{X}(\mu)}} \leq 1 / 4, \quad \mu \geq \mu_{331},
$$

and the running equation we will be concerned is

$$
\frac{1}{\alpha_{X}(\mu)}=\left[1-4 \sin ^{2} \theta_{W}\left(M_{Z}\right)\right] \frac{1}{\alpha\left(M_{Z}\right)}+\frac{1}{2 \pi}\left(b_{1}-3 b_{2}\right) \ln \left(\frac{M_{Z}}{\mu_{331}}\right)+\frac{1}{2 \pi} b_{X}^{r} \ln \left(\frac{\mu_{331}}{\mu}\right)
$$


where $b_{X}^{r}$, with $r=A, B$, are given below by Eqs. (14) for Model A, or by Eqs. (15) for Model $\mathrm{B}$, respectively; with $\sin ^{2} \theta_{W}\left(M_{Z}\right)=0.2311, \alpha\left(M_{Z}\right)=1 / 128$ and $M_{Z}=91.188 \mathrm{GeV}[29]$.

The $b_{i}$ coefficients in Model A, with and without SUSY, when the degrees of freedom above $\mu_{331}$ are taken into account, are given by:

$$
\begin{aligned}
& b_{X}^{A}=24+N_{\rho}+N_{\chi}, \\
& b_{X, J}^{A}=10+N_{\rho}+N_{\chi}, \\
& b_{X}^{A(\text { susy })}=36+3\left(N_{\rho}+N_{\chi}\right), \\
& b_{X, J}^{A(\text { susy })}=15+3\left(N_{\rho}+N_{\chi}\right) .
\end{aligned}
$$

Similarly, for the case of Model B, we have

$$
\begin{aligned}
& b_{X}^{B}=20+N_{\rho}+N_{\chi}, \\
& b_{X, J}^{B}=6+N_{\rho}+N_{\chi}, \\
& b_{X}^{B(\text { susy })}=30+3\left(N_{\rho}+N_{\chi}\right), \\
& b_{X, J}^{B(\text { susy })}=9+3\left(N_{\rho}+N_{\chi}\right) .
\end{aligned}
$$

In Eqs. (14) and (15) we also show the cases when we omit the exotic quarks (this is denoted by,$J$ in $b_{X}$ ). We recall that in the supersymmetric versions we have assumed $\mu_{\text {susy }} \approx \mu_{331}$.

In the minimal non-SUSY version of models $\mathrm{A}$ and $\mathrm{B}$, we have $N_{\rho}=N_{\chi}=1$ and $N_{\rho}=N_{\chi}=2$ in the respective SUSY models. Notice that in both models adding more triplet of scalars like $\rho$ and $\chi$ enhance $b_{X}$ and produce a lower value for $\Lambda$. In the SUSY version the running is always faster.

\section{THE LANDAU POLE}

We see from Eq. (12) that $\alpha_{X}(\mu) \rightarrow \infty$ when $\mu \rightarrow \Lambda$, and we have $\sin ^{2} \theta_{W}(\mu) \rightarrow 0.25$. This is the Landau pole that we have mentioned in Sec. II In practice we study, using Eq. (13), what is the energy $\mu=M^{\prime}$ at which $\alpha_{X}\left(M^{\prime}\right)>1$, i.e., the condition when $\alpha_{X}$ becomes non-perturbative and we compare this result with the energy $\Lambda$ calculated directly by the expression

$$
\Lambda=\mu_{331} \exp \left(\frac{2 \pi}{b_{X} \alpha_{X}\left(\mu_{331}\right)}\right)
$$


which must coincide with, or be of the same order, the value obtained by using the condition $\sin ^{2} \theta_{W}(\Lambda)=0.25$. Of course, we expect that $M^{\prime} \lesssim \Lambda$.

\section{A. The Landau pole in Model A}

As we said before, at energies below $\mu_{331}$ there is an approximated $S U(3)_{C} \otimes S U(2)_{L} \otimes$ $U(1)_{Y}$ symmetry with the particle content of the SM plus a second scalar doublet, $i$. e., in the context of Model A: in the fermion sector there are the usual doublets $\left(\nu_{a}, l_{a}\right)_{L} \sim(\mathbf{1}, \mathbf{2},-1)$ and $\left(u_{\alpha}, d_{\alpha}\right)_{L} \sim(\mathbf{3}, \mathbf{2}, 1 / 3)$; the singlets $l_{a R} \sim(\mathbf{1}, \mathbf{1},-2)$ (and right-handed neutrinos but they do not affect the running of the constants); and $u_{\alpha R} \sim(\mathbf{3}, \mathbf{1}, 4 / 3), d_{\alpha R} \sim(\mathbf{3}, \mathbf{1},-2 / 3)$. In the scalar sector we have two doublets $\left(\eta^{0}, \eta_{1}^{-}\right) \sim(\mathbf{1}, \mathbf{2},-1)$ and $\left(\rho^{+}, \rho^{0}\right) \sim(\mathbf{1}, \mathbf{2},+1)$.

These are the degrees of freedom that are active at energies $\mu \leq \mu_{331}$. Hence, we have $N_{H}=2, N_{T}=0$ in the non-SUSY model and $N_{H}=4, N_{T}=0$ in the SUSY one. With this particle content we have from Eqs.(10) [for completeness we include the coefficient $b_{3}$ ]:

$$
\begin{aligned}
& \left(b_{1}, b_{2}, b_{3}\right)=(7,-3,-7), \\
& \left(b_{1}, b_{2}, b_{3}\right)^{\text {susy }}=(22 / 3,-8 / 3,-7),
\end{aligned}
$$

and using Eqs. (111) for obtaining $\alpha_{X}\left(\mu_{331}\right)$. If we use only the degrees of freedom that were used in obtaining the coefficients above we get, using Eq. (11), that $\sin ^{2} \theta_{W}(\Lambda)=0.25$ when $\Lambda \approx 4.10 \mathrm{TeV}$ in the non-SUSY case and $\Lambda \approx 4.8 \mathrm{TeV}$ in the SUSY case, as can be seen from Fig. 1]

Above the $\mu_{331}$ scale, the full representation of the 3-3-1 model have to be taken into account and we get, according to Eqs.(14) [for future use we have included the coefficients $\left.b_{L}, b_{3}\right]$,

$$
\begin{aligned}
& \left(b_{X}, b_{L}, b_{3}\right)^{A}=(26,-13 / 2,-5), \\
& \left(b_{X}, b_{L}, b_{3}\right)_{j}^{A}=(12,-13 / 2,-7) ; \\
& \left(b_{X}, b_{L}, b_{3}\right)^{A(\text { susy })}=(48,0,0), \\
& \left(b_{X}, b_{L}, b_{3}\right)_{j}^{A(\text { susy })}=(27,0,-3) .
\end{aligned}
$$

With the exotic quarks we will consider two situations. First, that they have masses below $\Lambda$ and are taken into account in the evolution equations in the interval $\left[\mu_{331}, \Lambda\right]$; second, we 


\begin{tabular}{||c|c|c|c|c||}
\hline$\mu_{331}$ & $\hat{\alpha}_{X}\left(\mu_{331}\right)$ & $M^{\prime}$ & $\Lambda$ & $M_{Z^{\prime}}\left(\mu_{331}\right)$ \\
\hline 2.0 & 0.55 & $2.4(3.0)$ & $3.1(5.2)$ & 5.2 \\
1.5 & 0.39 & $2.2(3.4)$ & $2.8(5.7)$ & 3.3 \\
1.0 & 0.28 & $1.9(3.9)$ & $2.4(6.5)$ & 1.9 \\
0.75 & 0.23 & $1.7(4.2)$ & $2.1(7.1)$ & 1.3 \\
0.5 & 0.19 & $1.4(4.8)$ & $1.8(8.2)$ & 0.8 \\
\hline
\end{tabular}

TABLE I: Values of $M^{\prime}$ and $\Lambda$ for the non-SUSY Model A. The number inside parenthesis are the values for the case when we omit the exotic quarks in the running equation. We show, in the last column, an estimative for the mass of the $Z^{\prime}$ vector boson. All masses are in TeV.

\begin{tabular}{||c|c|c|c|c||}
\hline$\mu_{331}$ & $\hat{\alpha}_{X}\left(\mu_{331}\right)$ & $M^{\prime}$ & $\Lambda$ & $M_{Z^{\prime}}\left(\mu_{331}\right)$ \\
\hline 2.0 & 0.47 & $2.3(2.6)$ & $2.6(3.3)$ & 4.9 \\
1.5 & 0.35 & $1.9(2.3)$ & $2.1(2.9)$ & 3.1 \\
1.0 & 0.26 & $1.4(1.9)$ & $1.6(2.4)$ & 1.8 \\
0.75 & 0.22 & $1.2(1.7)$ & $1.3(2.1)$ & 1.2 \\
0.50 & 0.18 & $0.9(1.4)$ & $1.0(1.8)$ & 0.75 \\
\hline
\end{tabular}

TABLE II: Same as Table I but for the SUSY Model A.

assume that their masses are higher than $\Lambda$ and are not considered in the running coupling constants.

The result for the non-supersymmetric model appear in Table I for different values for the $\mu_{331}$ scale: $2.0,1.5,1.0,0.75$ and $0.50 \mathrm{TeV}$. The same is done in Table II for the supersymmetric model. In both cases the $M^{\prime}$ and $\Lambda$ values when the exotic quarks are considered heavy until the Landau pole scale are shown in parenthesis in the respective table. In the last column we show an order of magnitude of the $Z^{\prime}$ neutral vector boson (see below).

\section{B. The Landau pole in Model B}

In this case below $\mu_{331}$ in the scalar sector we have to consider three doublets $\left(\eta^{0}, \eta_{1}^{-}\right) \sim$ $(\mathbf{1}, \mathbf{2},-1),\left(\rho^{+}, \rho^{0}\right),\left(h_{2}^{+}, \sigma_{2}^{0}\right) \sim(\mathbf{1}, \mathbf{2},+1)$; and one non-hermitian triplet $T \sim(\mathbf{1}, \mathbf{3},+2)$. With this particle content we have from Eqs.(10), below $\mu_{331}$ i.e., we have $N_{H}=3$ and $N_{T}=1$ 


\begin{tabular}{||c|c|c|c|c||}
\hline$\mu_{331}$ & $\hat{\alpha}_{X}\left(\mu_{331}\right)$ & $M^{\prime}$ & $\Lambda$ & $M_{Z^{\prime}}\left(\mu_{331}\right)$ \\
\hline 2.0 & 0.40 & $3.0(6.3)$ & $4.0(13.9)$ & 4.5 \\
1.5 & 0.32 & $2.7(8.0)$ & $3.8(17.7)$ & 3.0 \\
1.0 & 0.24 & $2.4(11.3)$ & $3.2(24.8)$ & 1.7 \\
0.75 & 0.21 & $2.2(14.4)$ & $2.9(31.5)$ & 1.2 \\
0.5 & 0.17 & $1.9(20.1)$ & $2.5(44.2)$ & 0.7 \\
\hline
\end{tabular}

TABLE III: Same as Table I but for the Model B.

$\left(N_{H}=6, N_{T}=2\right.$ in the SUSY case):

$$
\begin{aligned}
& \left(b_{1}, b_{2}, b_{3}\right)=(49 / 6,-13 / 6,-7), \\
& \left(b_{1}, b_{2}, b_{3}\right)^{\text {susy }}=(32 / 3,-1,-7) .
\end{aligned}
$$

If we use only the degrees of freedom that were used in obtaining the coefficients above we get again, from Eq. (11), that $\sin ^{2} \theta_{W}(\Lambda)=0.25$ when $\Lambda \approx 5.7 \mathrm{TeV}$ in the non-SUSY case and $\Lambda \approx 7.8 \mathrm{TeV}$ in the SUSY case, as can be seen from Fig. 1. If we consider the doublet $\left(h_{2}^{+}, \sigma_{2}^{0}\right)$ heavy (but keeping its VEV small) we get $b_{1}=8$ and $b_{1}^{\text {susy }}=28 / 3$ and the values for $M^{\prime}$ and $\Lambda$ are a little bit smaller than the case considered here.

Above the $\mu_{331}$ scale, the full representation of the 3-3-1 model have to be taken into account and we obtain, according to Eqs.(15) [again for future use we have included again the coefficients $\left.b_{L}, b_{3}\right]$,

$$
\begin{aligned}
& \left(b_{X}, b_{L}, b_{3}\right)^{B}=(22,-17 / 3,-5), \\
& \left(b_{X}, b_{L}, b_{3}\right)_{y}^{B}=(8,-17 / 3,-7), \\
& \left(b_{X}, b_{L}, b_{3}\right)^{B \text { (susy) }}=(42,5,0), \\
& \left(b_{X}, b_{L}, b_{3}\right)_{\jmath}^{B \text { (susy) }}=(21,5,-3) .
\end{aligned}
$$

The results are shown in Table III for the non-SUSY Model B and in Table IV for the respective SUSY model.

\section{V. $\quad Z^{\prime}$ MASS AND 2-LOOP EVOLUTION EQUATIONS}

The $Z^{\prime}$ is the heaviest vector boson of the models, the Landau pole energy scale $(\Lambda)$ is supposed to be an upper limit for its mass in the context of a perturbative approach [25]. 


\begin{tabular}{||c|c|c|c|c||}
\hline$\mu_{331}$ & $\hat{\alpha}_{X}\left(\mu_{331}\right)$ & $M^{\prime}$ & $\Lambda$ & $M_{Z^{\prime}}\left(\mu_{331}\right)$ \\
\hline 2.0 & 0.34 & $2.7(3.5)$ & $3.1(4.8)$ & 4.1 \\
1.5 & 0.28 & $2.2(3.2)$ & $2.5(4.4)$ & 2.8 \\
1.0 & 0.22 & $1.7(2.8)$ & $1.9(3.8)$ & 1.7 \\
0.75 & 0.19 & $1.4(2.5)$ & $1.6(3.4)$ & 1.1 \\
0.5 & 0.17 & $1.0(2.2)$ & $1.2(3.0)$ & 0.7 \\
\hline
\end{tabular}

TABLE IV: Same as Table II, but for the SUSY Model B.

However, the mass of this boson, at the scale $\mu_{331}$, and assuming $\left\langle\chi^{0}\right\rangle \approx \mu_{331}$, has an order of magnitude given by

$$
M_{Z^{\prime}}\left(\mu_{331}\right) \simeq\left[4 \pi \alpha_{X}\left(\mu_{331}\right)\right]^{1 / 2} \mu_{331}
$$

The values for the estimative of $M_{Z^{\prime}}$ using Eq. (21) are shown in the last column of Tables 【IV. We see that for some values of $\alpha_{X}, M_{Z^{\prime}}$ is larger than $M^{\prime}$ or $\Lambda$.

It is interesting to note that in the SUSY version of Model A at energies above $\mu_{331}$ the dependence with the energy in $S U(3)_{L}$ and $S U(3)_{C}$ is lost since, as can be seen from Eq. (18), at the 1-loop we have that $b_{L}=b_{3}=0$, i.e.,

$$
\alpha_{L}\left(\mu>\mu_{331}\right)=\alpha_{3}\left(\mu>\mu_{331}\right)=\text { constant }
$$

and the same occurs for SUSY Model B for $\alpha_{3}$ as shown in Eq. (20). We can wonder if this is an artifact of the 1-loop approximation. Thus, let us consider the 2-loop evolution equations that are given by

$$
\mu \frac{d \alpha_{i}(\mu)}{d \mu}=\frac{1}{2 \pi}\left[b_{i}+\frac{1}{4 \pi} \sum_{j=1}^{3} b_{i j} \alpha_{j}(\mu)\right] \alpha_{i}(\mu)^{2},
$$

where we have not considered the Yukawa couplings since in any case their dominant contributions seems to be positive. For example, in SUSY Model A [30]

$$
b_{i j}=\left(\begin{array}{ccc}
\frac{784}{3}+12\left(N_{\rho}+N_{\chi}\right) & 32+16\left(N_{\rho}+N_{\chi}\right) & 160 \\
4+2\left(N_{\rho}+N_{\chi}\right) & 14+\frac{17}{3}\left(N_{\rho}+N_{\chi}\right) & 48 \\
\frac{62}{3} & 24 & 48
\end{array}\right) .
$$

In fact, in Eqs. (23), we have $N_{\rho}=N_{\chi}=2$ since we are considering the SUSY version. We see that even at this order the asymptotic freedom for QCD has been lost for energies higher 
than $\mu_{331}$. The lose of the asymptotic freedom at higher energies, for both the SUSY Model $\mathrm{A}$ and $\mathrm{B}$ is a prediction of the models since this result does not depend on the value of $\mu_{331}$. Of course, a more careful analysis should be done.

\section{DISCUSSIONS}

We have re-examined the question of the non-perturbative limit and the Landau-like pole in 3-3-1 models. In addition we have considered the respective supersymmetric versions and also the situation when the exotic quarks are heavy enough and do not enter in the running equation of $\alpha_{X}$. In practice what we have studied is the energy scale at which a model loses its perturbative character, $M^{\prime}$, or calculated directly the Landau pole, $\Lambda$, from Eq. (16). We find, as expected, that for all these models these energy scales are of the same order of magnitude, i.e., $M^{\prime} \lesssim \Lambda$.

From Table 1 we see that for Model A, the values of $M^{\prime}$ and $\Lambda$ decrease with the value of $\mu_{331}$ but increase for lower $\mu_{331}$ if we omit the exotic quarks in the running equation. The maximal values of $4.8 \mathrm{TeV}$ or $8.2 \mathrm{TeV}$ without the exotic quarks, respectively, occur when $\mu_{331}=500 \mathrm{GeV}$. For the respective SUSY cases, we see from Table Ithat $M^{\prime}$ and $\Lambda$ always decrease with $\mu_{331}$ and also that they have lower values than the respective non-SUSY model. The result for the model with the scalar sextet (Model B) are shown in Tables III and IV. The largest value for $M^{\prime}(\Lambda)$ is 20.1 (44.2) TeV when the heavy quarks are not considered. As in Model A, both scales also decrease with the value of $\mu_{331}$.

Notice that from Table 【 the value of $\Lambda$ (or $M^{\prime}$ ) for Model A (without SUSY) is always lower that the value obtained in Refs. 25, 26, 27] and in Fig. 1. As we have mentioned before, the latter value should be an upper limit for $\Lambda$. This is confirmed when the extra degrees of freedom of the 3-3-1 model are taken into account, for energies above $\mu_{331}$. As said before, we can see from Tables 【IV that the value of $\Lambda$ increases when the scale $\mu_{331}$ increases. But, as $\mu_{331}$ becomes larger the difference between both energy scales becomes smaller and in some point $\mu_{331}$ must be equal to $\Lambda$. However, notice also that when we omit from the analyses the exotic quarks this upper bound is evaded. This happens because the right-handed components of those quarks have the largest value of the $U(1)_{X}$ charge making that $\alpha_{X}$ run more rapidly compared to the case where only the standard model particles are taken into account. When the heavy quarks are switch off $\alpha_{X}$ run again slowly and the 
Landau-like pole occurs at a higher energy. The scenarios without the exotic quarks could be realized if there are strong dynamical effects with these degrees of freedom in this range of energy an probably the number of scalar multiplets of these models may be lower than it has been considered [31]. Notice also that since SUSY implies more degrees of freedom the values of $M^{\prime}$ and $\Lambda$ are always lower than in the respective non-SUSY model. If the Landau pole is calculated by using only the degrees of freedom below the 3-3-1 energy and the condition $\sin ^{2} \theta_{W}(\Lambda)=0.25$ from Eq. (11), the value obtained is shown in Fig. 11, Since the value of $\mu_{331}$ is below of these values we have studied how the value of the pole and the perturbative limit are modified when $0.5 \leq \mu_{331} \leq 2 \mathrm{TeV}$.

Finally, let us mention that there is another type of 3-3-1 model in which the righthanded neutrinos or heavy neutral leptons belong to the same triplet than the ordinary leptons [32, 33]. The charge operator is defined in this case as

$$
\mathcal{Q}_{B}=\frac{1}{2}\left(\lambda_{3}-\frac{1}{\sqrt{3}} \lambda_{8}\right)+X .
$$

In this sort of models the Landau pole arise above the Planck scale and for this reason it has no physical consequences. However, models with electric charge operator defined by Eqs. (21) and (25) are embedded in an $S U(3)_{C} \otimes S U(4)_{L} \otimes U(1)_{N}$ but in this 3-4-1 model the equation relating the coupling constant $g_{L}$ and $g_{X}$ is given also by Eq. (11) [34]. Thus, our results are also valid for the case of 3-4-1 models.

A. G. D. was supported by FAPESP under the process 01/13607-3, R.M. was supported by COLCIENCIAS and V. P. was partially supported by CNPq under the process 306087/88-0.

[1] F. Pisano and V. Pleitez, Phys. Rev. D 46 (1992) 410; R. Foot, O. F. Hernandez, F. Pisano and V. Pleitez, Phys. Rev. D 47 (1993) 4158; P. Frampton, Phys. Rev. Lett. 69 (1992) 2889.

[2] V. Pleitez and M. D. Tonasse, Phys. Rev. D 48, 2353 (1993).

[3] C. A. de S. Pires and O. Ravinez, Phys. Rev. D 58, 035008 (1998).

[4] P. B. Pal, Phys. Rev. D 52, 1659 (1995).

[5] A. G. Dias, V. Pleitez, and M. D. Tonasse, Phys. Rev. D 67, 095008 (2003); A. G. Dias and V. Pleitez, Phys. Rev. D 69, 077702 (2004); A. G. Dias, C. A. de S. Pires, and P. S. Rodrigues da Silva, Phys. Rev. D 68, 115009 (2003) and references therein. 
[6] J. C. Montero, V. Pleitez and M. C. Rodriguez, Phys. Rev. D 65, 035006 (2002); ibid D70, 075004 (2004), and hep-ph/0406299

[7] S. Dimopoulos and D. E. Kaplan, Phys. Lett. B531, 127 (2002).

[8] C. Csáki, J. Erlich, G. D. Kribs, and J. Terning, Phys. Rev. D 66, 075008 (2002).

[9] S. Dimopoulos, D. E. Kaplan, and N. Weiner, Phys. Lett. B534, 124 (2002); T. Li and W. Liao, Phys. Rev. B545, 147(2002);

[10] W. Chang and J. N. Ng, Phys. Rev. D 69, 056005 (2004).

[11] I. Gogoladze, Y. Mimura, and S. Nandi, Phys. Lett. B554, 81 (2003).

[12] R. Martinez, W. A. Ponce, and L. A. Sánchez, Phys. Rev. D 65, 055013 (2002); W. A. Ponce, Y. Giraldo, and L. A. Sánchez, Phys. Rev. D 67 (2003) 075001.

[13] J. C. Montero, V. Pleitez, and M. C. Rodriguez, Phys. Rev. D 58, 094076, 097505 (1998), Int. J. Mod. Phys. A16, 1147, 2001; and hep-ph/0204130, P. H. Frampton and A. Rasin, Phys. Lett. B482, 129 (2000).

[14] M. A. Perez, G. Tavares-Velasco and J. J. Toscano, Phys. Rev. D 69, 115004 (2004).

[15] L. Willmann it et. al., Phys. Rev. Lett. 82, 49 (1999).

[16] V. Pleitez, Phys. Rev. D 61, 057903 (2000).

[17] B. Dion, T. Gregoire, D. London, L. Marleau and H. Nadeau, Phys. Rev. D 59, 075006 (1999).

[18] M. B. Tully and G. C. Joshi, Phys. Lett. B466, 333 (1999); E. M. Gregores, A. Gusso and S. F. Novaes, Phys. Rev. D 64, 015004 (2001); J. L. Garcia-Luna, G. Tavares-Velasco, J. J. Toscano, Phys. Rev. D 69, 093005 (2004).

[19] L. D. Landau, in Niels Bohr and the Development of Physics, edited by W. Pauli (Pergamon Press, London, 1955); p. 52, and references therein.

[20] M. Göckerler, et al., Phys. Rev. Lett. 19, 4119 (1998).

[21] H. Gies and J. Jaeckel, hep-ph/0405183

[22] S. Weinberg, The Quantum Theory of Fields, (Cambridge University Press, New York, 1996); Sec. 18.3.

[23] R. Dashen and H. Neuberger, Phys. Rev. Lett. 24, 1897 (1983); M. A. B. Bég, C. Panagiotakopoulos and A. Sirlin, Phys. Rev. Lett. 52, 883 (1984); M. Lindner, Z. Phys. C31, 295 (1986); J. Kuti, L. Lin and Y. Shen, Phys. Rev. Lett. 61, 678 (1988); P. Hasenfratz and J. Nager, Z. Phys. C 37, 477 (1988); M. Lüscher and P. Weisz, Phys. Lett. B212, 472 (1988); T. Hambye and K. Riesselmann, Phys. Rev. D, 55, 7255 (1997), and references therein. 
[24] K. Holland, hep-lat/0409112.

[25] D. Ng, Phys. Rev. D 49, 4805 (1994).

[26] P. Jain and S. D. Joglekar, Phys. Lett. B407, 151 (1997).

[27] P. H. Frampton, Int. J. Mod. Phys. A13, 2345 (1998). More recently a value of $4 \mathrm{TeV}$ for the energy of the pole has been given in P. H. Frampton, Mod. Phys. Lett. A18, 1377 (2003).

[28] J. C. Montero, V. Pleitez and O. Ravinez, Phys. Rev. D 60, 076003 (1999).

[29] S. Eidelman et al. (Particle Data Group), Phys. Lett. B592, 1 (2004).

[30] D. R. T. Jones, Phys. Rev. D 25, 581 (1982); M. E. Machacek and M. T. Vaugh, Nucl. Phys. B222, 83 (1983).

[31] P. Das and P. Jain, Phys. Rev. D 69, 075001 (2000).

[32] J. C. Montero, F. Pisano, and V. Pleitez, Phys. Rev. D 47, 2918 (1993); R. Foot, H. N. Long, and T. A. Tran, Phys. Rev. D 50, R34 (1994); H. N. Long, ibid. D 54, 4691 (1996).

[33] G. Tavares-Velasco and J. J. Toscano, Phys. Rev. D70, 053006 (2004), hep-ph/0407047.

[34] F. Pisano and V. Pleitez, Phys. Rev. D 51, 3865 (1995). 